\title{
一种基于对抗学习和语义相似度的社交网络跨媒体 搜索方法
}

\author{
刘羽中，杜军平*，周南 \\ 北京邮电大学智能通信软件与多媒体北京市重点实验室, 北京 100876 \\ * 通信作者. E-mail: junpingdu@126.com \\ 收稿日期: 2019-06-05; 修回日期: 2019-07-26；接受日期: 2019-09-04; 网络出版日期: 2021-04-13 \\ 国家自然科学基金 (批准号: 61532006, 61772083, 61802028, 61877006) 资助项目
}

\begin{abstract}
摘要 社交网络蕴含着丰富的多媒体信息, 如何实现社交网络跨媒体信息的搜索已成为研究热点. 基 于深度学习的单一模态语义特征提取和学习在社交网络信息搜索上取得了较好的效果. 在跨模态信息 搜索时不同模态的数据特征不能直接比较, 因此不同模态之间的语义鸿沟是亟待解决的关键问题. 针 对上述问题, 本文提出了一种基于对抗学习和语义相似度的跨媒体搜索方法, 实现了文本和图像之间 的相互匹配、排序和搜索. 该方法使用对抗学习方法框架构建训练特征映射网络和模态判别网络, 其 中特征映射网络使用多维语义分布向量将不同模态的数据映射到同一语义空间中, 使得相同语义下的 不同模态数据在该空间距离小, 不同语义下相同模态数据距离大. 使用语义分布及相似度作为特征映 射网训练依据, 模态判别网络负责判定空间中不同数据的模态. 基于对抗学习交替训练两个网络, 使 得特征映射网络得到的数据和原数据语义一致, 并消除模态特性, 最终在同一空间内使用相似度来排 序并得到搜索结果. 实验结果表明本文提出的方法在文本和图像的相互搜索的 map 值比同类方法高, 并验证了该方法在社交网络安全话题数据上的有效性.
\end{abstract}

关键词 跨媒体搜索, 对抗学习, 语义相似度, 社交网络, 搜索排序

\section{1 引言}

随着移动互联网的高速发展, 社交网络数据信息呈现爆炸式增长 ${ }^{[1]}$, 越来越多的用户在各类社交 媒体上发布大量实时信息, 其中有关国民安全的信息显得尤为重要. 在事故灾害发生的时候, 能及时 搜索到和灾害相关的文本和图像并在一定程度上减小灾害带来的损失. 微博是社交网络重要的组成部 分, 具有内容短小、传播快、用户量大等特点. 微博对于国民安全相关热点事件内容具有敏感性, 因此 具有非常高的研究价值 ${ }^{[2,3]}$. 微博的数据形式不仅包括文本, 还有图像和视频, 单一媒体数据的搜索已

\footnotetext{
引用格式: 刘虫, 杜军平, 周南. 一种基于对抗学习和语义相似度的社交网络跨媒体搜索方法. 中国科学: 信息科学, 2021, 51: 779794, doi: 10.1360/SSI-2019-0120

Liu C, Du J P, Zhou N. A cross-media search method for social networks based on adversarial learning and semantic similarity (in Chinese). Sci Sin Inform, 2021, 51: 779-794, doi: 10.1360/SSI-2019-0120
} 
经不能很好地满足现有的社交网络信息需求, 社交网络尤其是基于微博的跨媒体信息搜索的需求与日 俱增.

近年来, 深度学习 ${ }^{[4]}$ 在文本和图像领域应用十分广泛, 它对数据特征的提取准确而高效, 为跨媒 体搜索提供了支持. 然而不同模态之间的数据特征分布不同, 语义空间不能互通, 即包含相同语义内 容的文本和图像不能通过它们所在的语义空间直接建立关联关系. 对抗学习 [5 8] 对于生成一个新的 数据分布非常有效, 已经被广泛应用于文本、图像、语音的生成 ${ }^{[9]}$.

本文提出一种基于对抗学习和语义相似度的社交网络跨媒体搜索方法 (semantic similarity based adversarial cross media retrieval, SSACR), 对国民安全相关的微博文本以及图像进行搜索, 并根据语义 相似度进行排序. 该方法主要使用对抗训练的方式训练两个神经网络模型, 即特征映射网络和模态判 别网络. 特征映射网络作为对抗学习的生成器分别将来自文本和图像的特征映射到同一语义空间中, 并使用语义及其相似度来训练特征映射网络, 相似度约束能够减小同语义下不同模态数据的差异. 模 态判别网络作为对抗学习判别器用来区分映射到同一语义空间中的数据的原本模态, 并以数据的真实 模态来训练模态判别网络. 本文提出的方法将搜索的文本或图像经过特征映射网投影到同一语义空间 后, 根据和该空间中其他数据的距离的远近来得到搜索结果排序.

本文提出的 SSACR 方法, 意在解决社交网络国民安全相关信息的跨媒体搜索问题,包括使用文 本搜索事故灾害相关图片, 以及使用事故照片查找相关文本信息等. 本文主要贡献是提出了一种基于 相似度比较的方法, 将映射到同一语义空间的不同模态数据的特征向量进行了相似度计算, 并与原本 的语义特征向量之间的相似度进行比较, 并以此作为特征映射网络的模态损失函数, 意在消除同一语 义下不同模态数据的差异, 解决了跨媒体数据之间的语义沟壑问题. 本文提出的 SSACR 方法, 使用所 有文本图象映射的特征向量的相似度矩阵代替了其他方法的文本图象二元组比较, 更加充分描述了跨 模态数据之间的关联, 同时减小了训练的复杂度. SSACR 方法使用了余弦相似度刻画数据的相关性, 而非其他方法的二值刻画, 使得语义描述更加精确. 最终使得跨媒体检索更加高效和准确.

\section{2 相关工作}

社交网络跨媒体搜索是近年来的研究热点, 深度学习方法已经在搜索结果准确率上取得重要突 破. 对抗学习通过生成网络和判别网络的交替迭代训练, 使得生成网络能够对目标特征分布进行有效 模拟, 在确保多样性的同时不失原有的数据特征.

\section{1 跨媒体搜索方法}

Feng 等 ${ }^{[10]}$ 通过关联两个单模自动编码器的隐藏表示来构建模型, 通过最小化每种模态误差的线 性组合以及两种模态之间的相关误差来训练模型, 最终实现搜索. 该方法对于数据量较小, 特征简单 的数据效果较好, 但是由于只关联模态线性组合, 对于特征较复杂的数据会欠拟合. Hardoon 等 ${ }^{[11]}$ 使 用核方法的相关性分析来学习 Web 图像及其相关文本的语义表示. 由于使用核方法, 抽取某个样本 特征需要计算和所有样本之间的核函数, 在数据量大的情况下训练速度较慢, 效率低, 适用于较小数 据量. Peng 等 ${ }^{[12]}$ 提出了跨媒体多深度网络 (cross-media multiple deep network), 利用复杂的跨媒体 关联通过两个阶段学习跨模态信息. 第 1 阶段学习同一模态和不同模态内的信息, 第 2 阶段训练跨模 态关联. 该方法主要分两步进行训练, 使得训练带来的误差进行累计, 因此到第 2 阶段的跨模态关联 的噪声比较大, 跨模态语义描述不准确. Wang 等 ${ }^{[13]}$ 提出一种将多模态数据映射到公共子空间的方 法, 可以测量不同模态数据之间的相似性, 并保留了模态间和模态内相似度关系. Wang 等 ${ }^{[14]}$ 提出了 
一种耦合线性回归框架来实现跨媒体搜索的方法, 并提出了一种基于半二次最小化的迭代算法来解决 正则化线性回归问题. 线性回归方法对于多种语义的数据拟合能力较弱, 不能刻画复杂的跨模态语义 关系, 因此适用于简单的数据.

$\mathrm{Li}$ 等 [15] 使用了线性相关模型研究不同的交叉模式关联方法, 提出了一种基于跨模态因子分析 (cross-modal factor analysis) 的跨模态关联方法实现跨媒体搜索. 该方法使用线性模型关联跨模态数 据, 对于不同模态数据的相关性刻画不充分, 适用于简单文本图象关联. Yao 等 [16] 提出了一种排名 典型相关分析 (ranking canonical correlation analysis) 来学习查询文本和图像相似性. 通过最大化查 询和图像之间的相关性来找到公共子空间. Andrew 等 ${ }^{[17]}$ 提出了深度典型相关分析 (deep canonical correlation analysis), 作为线性方法典型相关分析的非线性扩展. 以上两种方法基于 CCA (canonical correlation analysis) 方法, 仅考虑了成对文本图像的语义相关性, 对于同一模态下不同数据之间可能 存在的相关性并没有考虑, 语义描述不充分. Zhuang 等 ${ }^{[18]}$ 将耦合字典学习 (dictionary learning) 引 入到用于跨媒体搜索的监督稀疏编码中. 该方法基于字典学习, 对于数据源的噪声敏感, 对数据源要 求较高, 因此使用于数据较为干净的场景中. Ngiam 等 ${ }^{[19]}$ 提出了一系列多模式学习任务来学习多种 模态的特征, 并使用深层网络来学习交叉模态特征. 由于多模态特征较为复杂, 通过深层网络训练会 使得网络规模较大, 不适宜大规模数据训练. Yan 等 [20] 通过深度典型相关分析解决了在联合潜在空 间中匹配图像和文本的问题. 此方法用线性模型将不同媒体特征映射到同构的最大相关子空间, 不能 很好地挖掘跨媒体数据之间的联系. Song 等 ${ }^{[21]}$ 提出了一种新的跨媒体散列模型用于探索来自不同数 据源的多种跨媒体数据之间的相关性, 并通过 XOR 和位计数操作实现快速搜索. 此方法可以大量节 省内存空间, 但是散列的映射加大了训练的计算复杂度, 使得训练较慢. 同时在图象标签的语义建立 方面, $\mathrm{Li}$ 等 ${ }^{222}$ 提出了深度协作嵌入模型揭示了图像和标签的统一的潜在空间. 由于此种方法集成了 弱监督图象和标签的相关性, 因此对于社区贡献的数据有很好的适应性, 能解决部分标签数据缺失的 情况.

\section{2 对抗学习方法}

Wang 等 [23] 提出了一种对抗性跨模态搜索 (adversarial cross-modal retrieval) 方法, 它寻求有效 公共子空间的对抗性学习以及两个训练过程之间的相互作用. 第 1 个过程是特征映射过程, 它尝试在 公共子空间中生成模态不变的数据, 第 2 个过程是模态分类器, 它可以区分不同模态. 使用三联体约 束来对映射过程进行限制, 以尽量减少相同语义下不同模态数据的距离, 同时在语义上最大化不同的 图像和文本之间的距离. 此方法对于跨模态语义刻画比较准确, 引入三联体约束, 使用正负样本, 增 大了数据训练量, 使得训练速度较慢. $\mathrm{Xu}$ 等 ${ }^{[24]}$ 提出了一种新的深度对抗度量学习 (deep adversarial metric learning) 用于跨模态搜索, 并通过引入对抗性学习来增加额外的正则化. 此方法使用深度模型 进行特征提取, 使得特征描述比较丰富, 但是仅仅引入对抗学习增大跨模态搜索正则化, 对跨模态语 义描述还不够深刻, 使得模态间语义缺失. $\mathrm{He}$ 等 ${ }^{[25]}$ 提出了一种基于对抗性学习的新型无监督跨模态 搜索方法 (unsupervised cross-modal adversarial learning). 此方法基于无监督, 对于没有标注的数据比 较友好, 但是跨模态语义以及同模态的语义学习较为困难, 没有统一的语义作为训练的优化目标, 对于 复杂跨模态数据检索不适用. Li 等 ${ }^{[26]}$ 提出了一种自我监督的对抗性散列 (self-supervised adversarial hashing networks) 方法, 采用自我监督的方式将对抗性学习融入跨模态 Hash 中. Wu 等 ${ }^{[27]}$ 提出了 一种新的深度生成方法来进行跨模态搜索, 在没有配对训练样本的情况下通过循环一致性损失来学习 Hash 函数. 以上两种方法对于训练样本缺失的情况比较好, 不过深度生成方式以及自监督的训练较 难, 对于训练数据完备的情况不太适用. Zhang 等 ${ }^{[28]}$ 提出了一种无监督的生成对抗跨模态 Hash 方 
法 (unsupervised generative adversarial cross-modal hashing), 充分利用了对抗生成网络的可用性来进 行无监督学习来实现跨媒体搜索. 此方法使用 Hash 编码, 对于语义相关性的描述是二元的而不是数 值的, 对相关性描述偏两极化, 不能准确描述不同模态数据相似度的数值.

\section{SSACR 方法简介}

\subsection{SSACR 方法的描述}

本文以实现两个模态之间的相互搜索为基本目的. 定义跨媒体数据由 $n$ 个三元组构成的, 记为 $O=\left\{o_{i}\right\}_{i=1}^{n}, o_{i}=\left(v_{i}, t_{i}, l_{i}\right)$. 其中 $v_{i} \in \mathbb{R}^{d_{v}}$ 表示图像特征向量, 它的维度是 $d_{v}, t_{i} \in \mathbb{R}^{d_{t}}$ 表示文本特 征向量, 它的维度是 $d_{t}$. 其中, $d_{v} \neq d_{t}$, 语义向量 $l_{i}$ 表示这个文本图像对的语义分布, 即通过高维特 征向量形式化表示文本和图像的内容. 这个分布可以是简单的标签类别分布, 假设共有 $d_{l}$ 个类别. 对 于每个类别 $l_{i k}$, 如果这个图像 - 文本二元组属于这个类别, 则 $l_{i k}$ 为 1 , 否则为 0 . 同时也可以采用预 处理后的主题模型描述这个文本图像对的语义分布情况. 分别定义由所有 $o_{i}$ 构成的图像特征矩阵为 $V=\left\{v_{1}, \ldots, v_{n}\right\} \in \mathbb{R}^{d_{v} \times n}$, 构成的文本特征矩阵 $T=\left\{t_{1}, \ldots, t_{n}\right\} \in \mathbb{R}^{d_{t} \times n}$, 图像以及文本的语义矩阵 为 $L=\left\{l_{1}, \ldots, l_{n}\right\} \in \mathbb{R}^{d_{l} \times n}$.

为了统一图像和文本跨媒体语义, 引入特征映射网络, 将两种模态的数据经过特征映射网络统一 映射到公共语义空间 $S$ 中. 图像的特征映射表示为 $S_{V}=f_{V}\left(V ; \theta_{V}\right)$, 文本的特征映射表示为 $S_{T}=$ $f_{T}\left(T ; \theta_{T}\right)$. 其中 $f_{V}\left(V ; \theta_{V}\right)$ 为图像特征的映射函数, $f_{T}\left(T ; \theta_{T}\right)$ 为文本特征的映射函数, $\theta_{V}$ 和 $\theta_{T}$ 分别 表示图像特征网络和文本特征网络的参数. $S_{V}$ 和 $S_{T}$ 分别表示图像和文本特征映射在 $S$ 中的新特征, $S_{V}, S_{T} \in \mathbb{R}^{m \times n}$, 表示在公共语义空间 $S$ 中它们的新特征维度都是 $m$. 本文提出的 SSACR 方法在公 共语义空间 $S$ 中得到合适的 $S_{V}$ 和 $S_{T}$, 使得它们保持映射前的语义关系, 映射的性能由输入输出内 容的语义特征分布偏差决定, 并通过差值训练特征映射网络. 在维持语义不变的前提下, 使得语义相 近的不同模态的数据在 $S$ 中距离较近, 相同模态的不同语义的数据在 $S$ 中距离较远.

\subsection{SSACR 方法的组成}

本文提出的 SSACR 方法包括图像特征输入网络、文本特征输入网络、特征映射网络、语义分布 网络、语义相似网络, 以及模态判别网络. 对于每一个图像 - 文本 - 语义三元组, 先将图像和文本的 特征进行提取, 然后分别输入到图像特征映射网络和文本特征映射网络中, 语义向量输入到语义分布 网络中. 语义相似度网络对输入的语义向量进行相似度计算. 特征映射网络将图像和文本特征映射到 $S$ 中, 模态判别网络根据映射到 $S$ 数据的模态进行判别.

特征映射网络通过最小化同模态数据映射前后的语义偏差以及同语义数据映射后的模态偏差达 到最优. 模态判别网络通过最小化映射后数据原本模态判定的误差达到最优. 特征映射网络和模态判 别网络通过对抗学习的方式进行训练, 使得两个网络最终都达到较优结构. 总体流程如图 1 所示.

\section{4 基于 SSACR 方法的跨媒体对抗学习}

\section{1 特征映射网络}

特征映射网络分为两部分, 图像特征映射网络和文本特征映射网络, 它们负责把原始数据特征映 射到同一语义空间当中. 为了保证映射之后的数据维持原模态的语义特征, 在特征映射网后面加入一 个语义预测网络, 并将分类器 softmax 的输出作为结果用于预测映射到同一语义空间的数据的语义分 


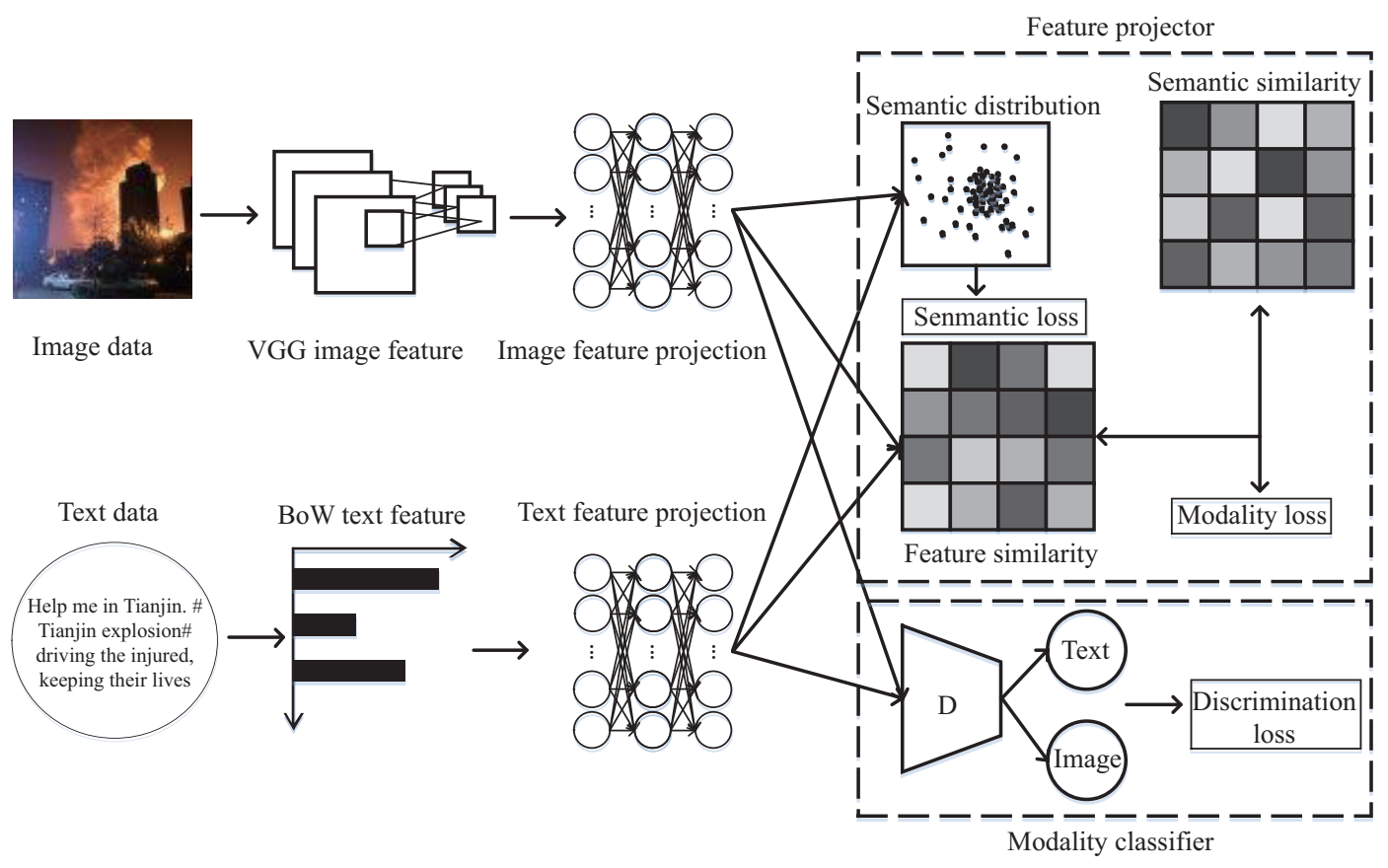

图 1 (网络版彩图) SSACR 方法的总体流程图

Figure 1 (Color online) The general flowchart of the proposed SSACR method

布. 令网络的参数为 $\theta_{\mathrm{imd}}$, 图像、文本两种模态中第 $i$ 个数据语义分布的第 $c$ 维值分别是 $p_{i c}\left(v_{i}\right)$ 和 $p_{i c}\left(t_{i}\right)$, 使用交叉熵计算新空间中语义的偏差值 $L_{\mathrm{imd}}$, 其表达式为

$$
L_{\mathrm{imd}}\left(\theta_{\mathrm{imd}}\right)=-\frac{1}{n} \sum_{i=1}^{n} \sum_{c=1}^{d_{l}}\left(y_{i c} \cdot\left(\log p_{i c}\left(v_{i}\right)+\log p_{i c}\left(t_{i}\right)\right)\right),
$$

其中 $L_{\mathrm{imd}}$ 计算在新的语义空间中每个新映射的数据和原来数据语义分布的差值, 包括图像和文本两 部分的差值总和, 目的是保证经过 $f_{V}\left(V ; \theta_{V}\right)$ 以及 $f_{T}\left(T ; \theta_{T}\right)$ 的转换使得原本同一模态中语义相近的 数据在新空间中距离依然相近, 语义较远的数据在新空间中距离依然较远.

为了保证不同模态下的数据经过特征映射后, 具有相同语义的不同模态的数据距离较近, 不同模 态的不同语义的数据相距较远, 在计算输入内容语义分布相似度的基础上, 使用原数据语义分布 $l_{1 \ldots n}$ 来构造语义相似度矩阵 $\operatorname{Sim}_{L} \in \mathbb{R}^{n \times n}$. 任意两个数据的语义分布分别是 $l_{a}$ 和 $l_{b}$, 它们的相似度定义为

$$
\operatorname{sim}\left(l_{a}, l_{b}\right)=\frac{l_{a i} \cdot l_{b i}}{\left\|l_{a i}\right\| \cdot\left\|l_{b i}\right\|}=\frac{\sum_{i=1}^{d_{l}} l_{a i} \times l_{b i}}{\sqrt{\sum_{i=1}^{d_{l}}\left(l_{a i}\right)^{2}} \times \sqrt{\sum_{i=1}^{d_{l}}\left(l_{b i}\right)^{2}}},
$$

这里需要对所有的分布 $l_{1 \ldots n}$ 进行相似度计算, 并得到所有数据下的语义相似性, $\operatorname{Sim}_{L}$ 的计算如下 所示:

$$
\operatorname{Sim}_{L}(i, j)=\operatorname{sim}\left(l_{i}, l_{j}\right),
$$

计算得到输入文本图象对之间的语义相似度后, 针对计算映射之后数据特征的相似度进行计算. 对于 任意两组数据 $o_{a}$ 和 $o_{b}$, 抽取 $o_{a}$ 的图像, 经过特征映射网后变成 $S_{v a}$. 从 $o_{b}$ 抽取文本, 经过特征映 射网络后变成 $S_{t b}$, 其相似度通过 $\operatorname{sim}\left(S_{v a}, S_{t b}\right)$ 来计算. 对整个数据集映射后的特征进行相似度求解, 
$\operatorname{Sim}_{S} \in \mathbb{R}^{n \times n}$ 即为得到的映射后数据特征相似度矩阵, 计算如下所示:

$$
\operatorname{Sim}_{S}(i, j)=\operatorname{sim}\left(s_{v i}, s_{t j}\right)=\operatorname{sim}\left(f_{V}\left(v_{i} ; \theta_{V}\right), f_{T}\left(t_{j} ; \theta_{T}\right)\right) .
$$

为保证不同模态之间的数据映射后的距离和它们表达的语义是相关的, 即距离较近的数据语义分布也 相近, 距离较远的数据语义分布也较远, 本文选用 $l_{2}$ 范数来描述两个相似度矩阵之间的差异, 并将差 异值定义为模态偏差值 $L_{\mathrm{imi}}\left(\theta_{V}, \theta_{T}\right)$, 计算如下所示:

$$
\begin{aligned}
L_{\mathrm{imi}}\left(\theta_{V}, \theta_{T}\right)= & \ell_{2}\left(\operatorname{Sim}_{L}, \operatorname{Sim}_{S}\right) \\
= & \sum_{i=1}^{n} \sum_{j=1}^{n}\left\|\operatorname{sim}\left(l_{i}, l_{j}\right)-\operatorname{sim}\left(f_{V}\left(v_{i} ; \theta_{V}\right), f_{T}\left(t_{j} ; \theta_{T}\right)\right)\right\|_{2} \\
= & \sum_{i=1}^{n} \sum_{j=1}^{n} \| \frac{\sum_{k=1}^{d_{l}} l_{i k} \times l_{j k}}{\sqrt{\sum_{k=1}^{d_{l}}\left(l_{i k}\right)^{2}} \times \sqrt{\sum_{k=1}^{d_{l}}\left(l_{j k}\right)^{2}}} \\
& -\frac{\sum_{k=1}^{m} f_{V}\left(v_{i} ; \theta_{V}\right)_{k} \times f_{T}\left(t_{j} ; \theta_{T}\right)_{k}}{\sqrt{\sum_{k=1}^{m}\left(f_{V}\left(v_{i} ; \theta_{V}\right)_{k}\right)^{2}} \times \sqrt{\sum_{k=1}^{d_{l}}\left(f_{T}\left(t_{j} ; \theta_{T}\right)_{k}\right)^{2}}} \|_{2} .
\end{aligned}
$$

将特征映射网络的总体损失函数定义为 $L_{\mathrm{emb}}$, 通过由语义偏差 $L_{\mathrm{imd}}$ 以及模态偏差 $L_{\mathrm{imi}}$ 的线性 加权求和构成. 其中 $\alpha$ 和 $\beta$ 分别表示两个偏差值对损失函数的贡献, 映射损失的计算如下所示:

$$
L_{\mathrm{emb}}\left(\theta_{V}, \theta_{T}, \theta_{\mathrm{imd}}\right)=\alpha \cdot L_{\mathrm{imd}}+\beta \cdot L_{\mathrm{imi}},
$$

$\alpha$ 和 $\beta$ 作为网络的超参数通过后续实验确定最优值.

\section{2 模态判别网络}

模态判别网络主要区分映射到公共语义空间的数据的原始模态. 令通过图像映射后的数据标签为 0 , 通过文本映射后的数据标签为 1 , 模态判别网络尽可能对数据的原始模态进行准确判断. 使用一个 神经网络进行计算, 并将该网络的损失函数定义为模态预测的偏差值. 损失函数 $L_{\mathrm{adv}}$ 计算如下所示:

$$
\begin{aligned}
L_{\mathrm{adv}}\left(\theta_{D}\right) & =-\frac{1}{n} \sum_{i=1}^{n} \log \left(1-D\left(s_{v i} ; \theta_{D}\right)\right)+\log \left(D\left(s_{t i} ; \theta_{D}\right)\right) \\
& =-\frac{1}{n} \sum_{i=1}^{n} \log \left(1-D\left(f_{V}\left(v_{i} ; \theta_{V}\right) ; \theta_{D}\right)\right)+\log \left(D\left(f_{T}\left(t_{i} ; \theta_{T}\right) ; \theta_{D}\right)\right),
\end{aligned}
$$

其中 $\theta_{D}, D\left(x, \theta_{D}\right)$ 表示该网络判定 $x$ 是文本的概率.

\section{3 对抗学习设计}

训练流程由两个网络结构的协同训练组成, 训练目标如式 (5) 和 (7) 所示. 基于对抗的思想优化 两个损失函数的值, 特征映射网络目标是尽可能保持语义消除模态, 模态判别网络的目标是尽可能在 公共语义空间中区分不同数据的模态, 特征映射网络和模态判别网络参数优化表达式如下所示:

$$
\begin{gathered}
\left(\theta_{v}, \theta_{T}, \theta_{\mathrm{imd}}\right)=\arg \min \left(L_{\mathrm{emb}}\left(\theta_{V}, \theta_{T}, \theta_{\mathrm{imd}}\right)-L_{\mathrm{adv}}\left(\theta_{D}\right)\right), \\
\theta_{D}=\arg \max \left(L_{\mathrm{emb}}\left(\theta_{V}, \theta_{T}, \theta_{\mathrm{imd}}\right)-L_{\mathrm{adv}}\left(\theta_{D}\right)\right) .
\end{gathered}
$$

具体训练过程如算法 1 所示. 


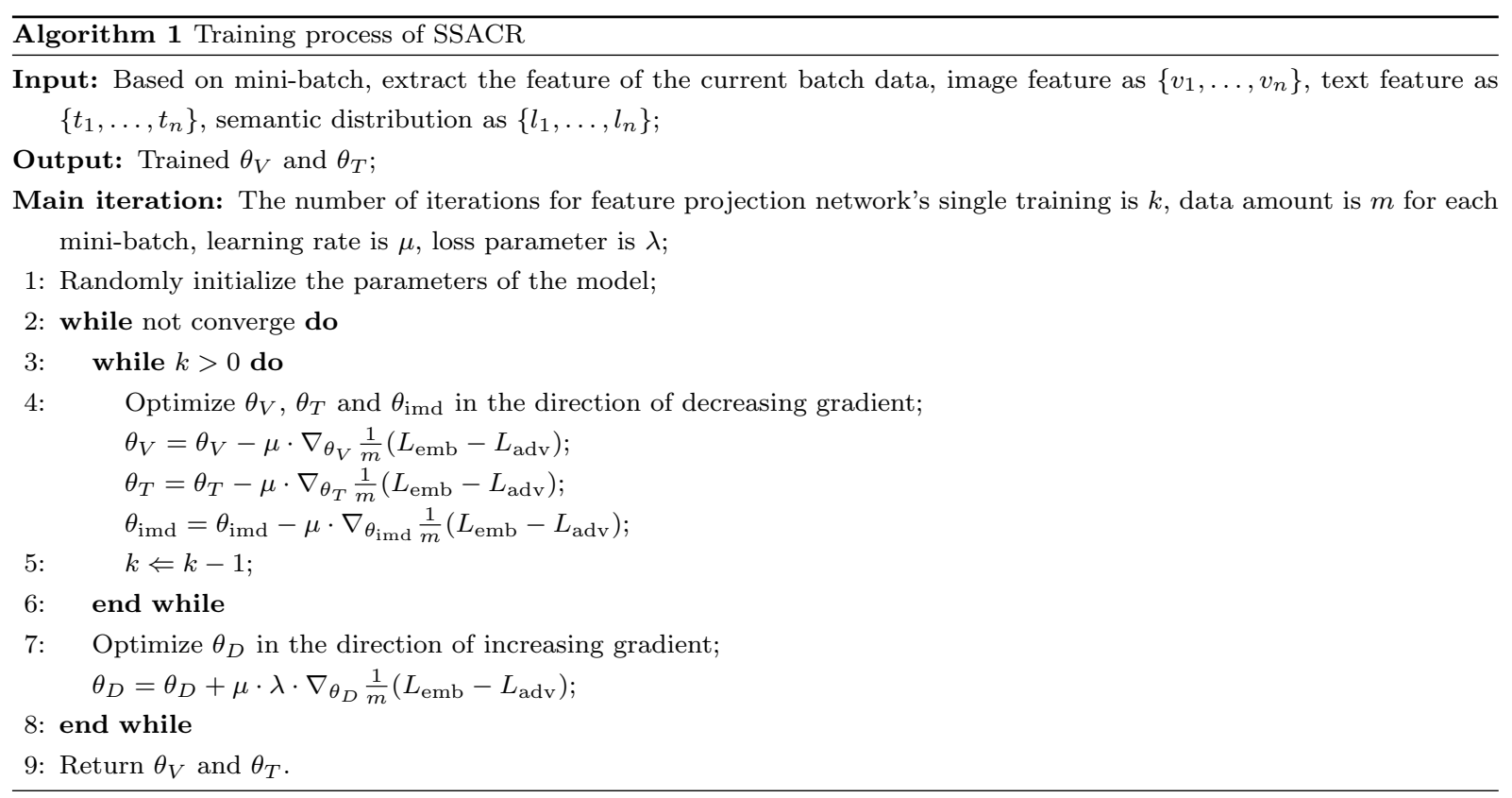

\section{4 搜索过程}

跨媒体搜索问题定义为给定一个新的搜索条目 $x$, 找到和 $x$ 不同模态的最相近的 top- $K$ 个结果. 搜索过程如算法 2 所示.

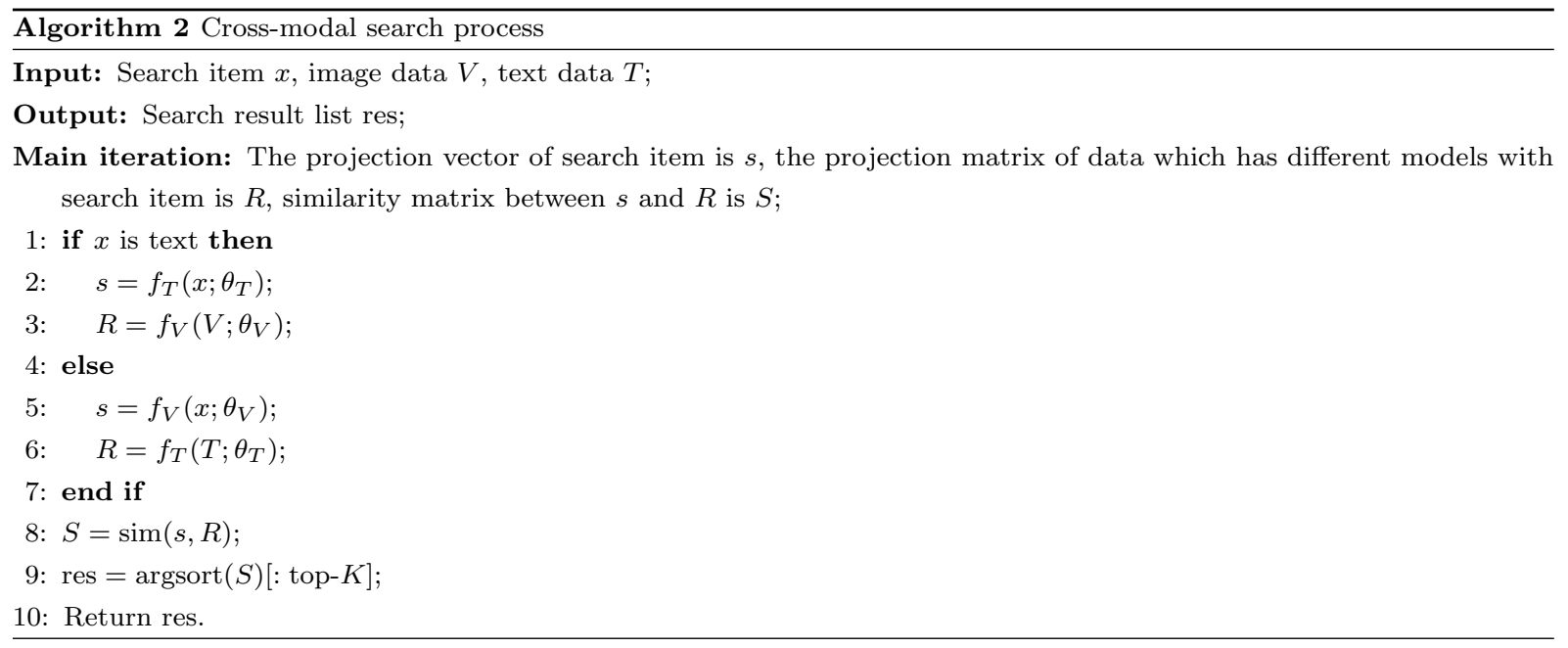

\section{5 实验结果与分析}

\section{1 评价指标}

实验使用采集自新浪微博的真实数据以及维基百科数据, 通过实验对本文提出的 SSACR 方法进 行验证, 并与其他算法在这两个数据集上进行对比. 本文使用 map (mean average precision) [29] 方法 评价跨媒体检索效果. map 共同考虑排名信息和排名精度, 是单个主题的平均精度的均值, 也就是每 
表 1 实验使用的数据集的统计信息

Table 1 Statistics of the datasets in our experiments

\begin{tabular}{cccccc}
\hline & Training instance & Test instance & Labels & Image feature & Text feature \\
\hline Wikipedia dataset & 2173 & 693 & 10 & 4096d VGG19 & $5000 \mathrm{~d}$ BoW \\
Weibo dataset & 4262 & 473 & 5 & 4096d VGG19 & $6739 \mathrm{~d}$ BoW \\
\hline
\end{tabular}

篇相关文档检索出后的精度的平均值. 它反映系统在全部相关文档或图象上性能的单值指标, 搜索系 统检索出来的相关文档越靠前, map 就可能越高, 反之越低.

本文也使用 PR 曲线 (precision recall curve) ${ }^{[30]}$ 度量跨媒体检索效果. PR 曲线描述的是检索中 精确率和召回率的关系, 本文度量的是精确率随召回率变化的曲线, 召回率作为横轴, 精确率作为纵 轴, 图上一点表示在当前召回率能取得的最大精确率. 在相同召回率下, 精确率越高, 说明没有检索出 更多不相关的文档, 检索越准确.

\section{2 实验设置}

\subsection{1 数据集}

微博数据集主要包含爆炸以及其他事件的 4735 个文本图像数据对, 其中爆炸事件相关数据占比 为 $30 \%$, 并将数据集中的 4262 对数据用作训练集, 473 对数据用作测试集. 维基百科数据集中总共有 2866 对数据, 其中 2173 对数据用作训练集, 693 对数据用作测试集, 数据以及特征的分布如表 1 所示.

\subsection{2 网络结构设计}

图像和文本特征映射网络都采用 3 层全连接神经网络结构, 并使用 $\tanh$ 作为激活函数, 将图像 和文本特征全部映射到公共语义空间中. 图像特征映射网络的每层神经元节点数分别是 $512,100,40$; 文本特征映射网络的每层神经元节点数分别是 $40,100,40$. 对于语义判别网络, 采用单层神经网络结 构, 神经元节点数是语义分布的维度, 对于模态判别网络, 采用 3 层全连接神经网络, 每层神经元节点 数分别是 $20,10,2$. 语义判别网络和模态判别网络采用 softmax 作为输出层.

\section{3 不同方法实验对比}

使用 map 来度量不同搜索方法的性能, 分别在维基数据集和微博数据集通过文本搜索图像和依 据图像搜索文本这两个任务的前 5 , 前 20 和前 50 个搜索结果计算 map 值. 对比方法在维基数据集和 微博数据集的结果分别如表 2 和 3 所示.

如表 2 和 3 所示, 提出的 SSACR 方法在维基公共数据集以及微博数据集上的 map 指标好于其 他方法.

由于 $\mathrm{CCA}$ 方法关注的是图片文本对的关联关系, 使用关联损失函数作为优化目标, 而忽略了文本 图像对之间内部元素的语义关联关系, 因此对于同种语义不同模态的数据不能建立充足的关联关系, 语义表达欠缺, 因此无论在图片搜索还是文本搜索方面的效果都远低于 SSACR 方法. JFSSL (joint feature selection and subspace learning) 方法虽然同样使用标签来拉大同模态不同语义元素之间的距离, 但是由于没有考虑不同模态下语义相似元素之间的距离, 不能很好地描述不同模态的相关性. CMDN (cross-media multiple deep networks) 方法使用深度神经网络来建立模型, 并用多任务训练方法优化同 模态不同语义元素距离和不同模态相同语义元素距离. SSACR 方法使用对抗学习的方法使得语义映 射网络达到更优的结构, 因此效果比 CMDN 方法好. ACMR (adversarial cross-modal retrieval) 方法使 


\section{表 2 跨媒体搜索方法在维基数据集的性能比较}

Table 2 Comparison of cross-media retrieval performance on Wikipedia dataset

\begin{tabular}{|c|c|c|c|c|c|c|c|c|c|}
\hline & \multicolumn{3}{|c|}{ map@5 } & \multicolumn{3}{|c|}{ map@20 } & \multicolumn{3}{|c|}{ map@50 } \\
\hline & txt2img & img2txt & Average & txt2img & img2txt & Average & txt2img & img2txt & Average \\
\hline $\mathrm{CCA}$ & 0.2685 & 0.2151 & 0.2418 & 0.2831 & 0.2209 & 0.2520 & 0.2543 & 0.2178 & 0.2361 \\
\hline JFSSL & 0.4406 & 0.3473 & 0.3940 & 0.4264 & 0.3576 & 0.3920 & 0.4146 & 0.3454 & 0.3800 \\
\hline CMDN & 0.5094 & 0.4125 & 0.4609 & 0.4895 & 0.4102 & 0.4498 & 0.4624 & 0.3956 & 0.4290 \\
\hline ACMR & 0.6225 & 0.4987 & 0.5606 & 0.6109 & 0.4986 & 0.5548 & 0.5732 & 0.4835 & 0.5284 \\
\hline DSCMR & 0.6342 & 0.4982 & 0.5662 & 0.6421 & 0.5012 & 0.5716 & 0.6347 & 0.4901 & 0.5624 \\
\hline Ours & 0.6604 & 0.4964 & 0.5784 & 0.6647 & 0.5052 & 0.5850 & 0.6436 & 0.4964 & 0.5700 \\
\hline
\end{tabular}

表 3 跨媒体搜索方法在微博数据集的性能比较

Table 3 Comparison of cross-media retrieval performance on Weibo dataset

\begin{tabular}{|c|c|c|c|c|c|c|c|c|c|}
\hline & \multicolumn{3}{|c|}{ map@5 } & \multicolumn{3}{|c|}{ map@20 } & \multicolumn{3}{|c|}{ map@50 } \\
\hline & txt2img & img2txt & Average & txt2img & img2txt & Average & txt2img & img2txt & Average \\
\hline CCA & 0.3885 & 0.3251 & 0.3568 & 0.3583 & 0.3239 & 0.3411 & 0.3213 & 0.3199 & 0.3206 \\
\hline JFSSL & 0.6478 & 0.5351 & 0.5915 & 0.6128 & 0.5181 & 0.5655 & 0.5197 & 0.5282 & 0.5239 \\
\hline CMDN & 0.7183 & 0.5814 & 0.6499 & 0.6799 & 0.5843 & 0.6321 & 0.5906 & 0.5729 & 0.5817 \\
\hline ACMR & 0.8653 & 0.7133 & 0.7893 & 0.8238 & 0.7071 & 0.7655 & 0.7065 & 0.6992 & 0.7029 \\
\hline DSCMR & 0.8742 & 0.7177 & 0.7960 & 0.8192 & 0.7246 & 0.7719 & 0.7410 & 0.7267 & 0.7339 \\
\hline Ours & 0.8792 & 0.7344 & 0.8068 & 0.8161 & 0.7361 & 0.7761 & 0.7493 & 0.7410 & 0.7452 \\
\hline
\end{tabular}

用对抗学习的方法对于同模态和不同模态数据距离都进行优化, 它采用三元组约束的方法来降低不同 模态数据相同语义的距离. 本文提出的 SSACR 方法使用语义相似度描述不同模态之间语义的距离. 因为三元组的构成是二元地判断文本和图像是否有关, 而实际上文本和图像的相关性并不能简单用 0 和 1 表示, 而用实数表达更为准确, 因此 SSACR 方法效果优于 ACMR 方法. DSCMR (deep supervised cross-modal retrieval) ${ }^{[31]}$ 使用 TextCNN 对文本特征进行抽取, 并构建深度特征映射网络将图象和文 本特征映射到同一语义空间, 但是由于特征映射网络模块过重, 容易导致训练过拟合, 因此使用基于 相似度的 SSACR 方法效果稍好.

在微博数据集上本文方法和对比方法的效果和维基数据相似且表现比较稳定, 评价指标在整体上 有所提高, 原因是微博数据集在语义特征分布上相对简单, 跨媒体搜索任务难度有所降低. 本文对比 了 SSACR 和 ACMR 方法在文本搜索图片以及图片搜索文本的结果. 在维基数据集进行实验所得出 的 PR 曲线和在微博数据集进行实验所得出的 PR 曲线分别如图 2 和 3 所示.

如图 2 和 3 所示, 在文本搜索图像方面, 当召回率较高的情况下 SSACR 方法准确率略高于 ACMR. 从 PR 曲线的走势来看, 在维基数据集上,在召回率持续增高的情况下, SSACR 方法的准确率下降较 慢, 维持一个相对较高水平, ACMR 准确率迅速下降并稳定, 这说明 SSACR 方法对搜索的结果稳定 性较高, 优于 ACMR 方法. 本文方法在微博数据集上所得到的 PR 曲线分布和维基数据集相似, 说明 SSACR 方法在召回率和准确率的整体分布上要优于 ACMR 方法. 

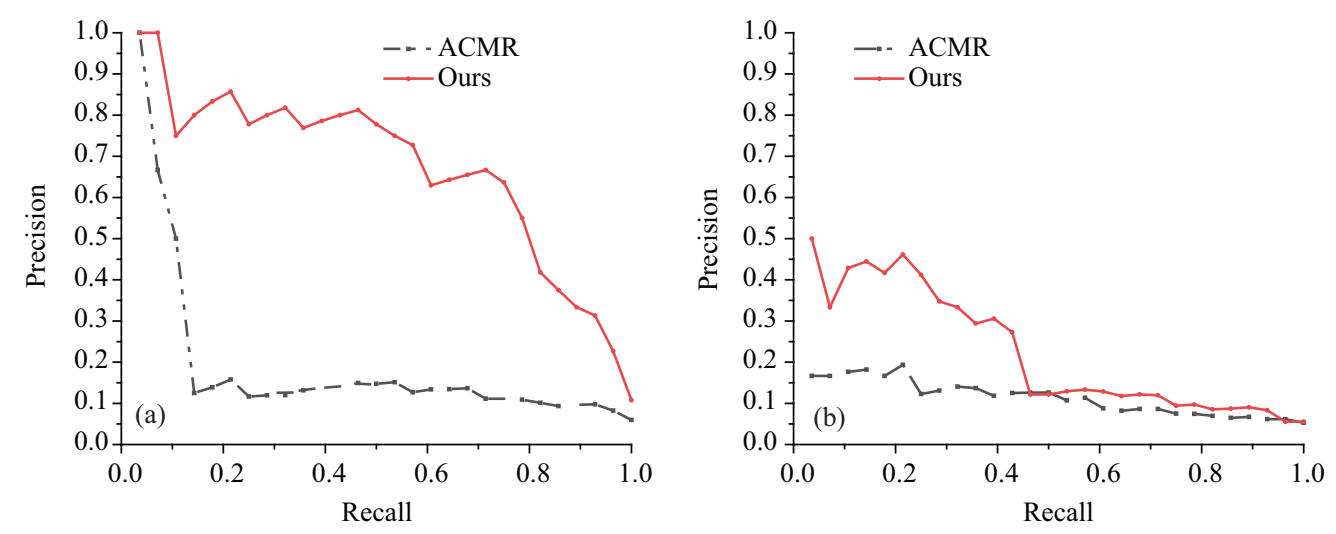

图 2 (网络版彩图) 图到文本 (a), 文本到图 (b) 搜索的 PR 曲线结果/维基数据集

Figure 2 (Color online) PR curve of image to text (a), text to image (b) search/Wikipedia dataset
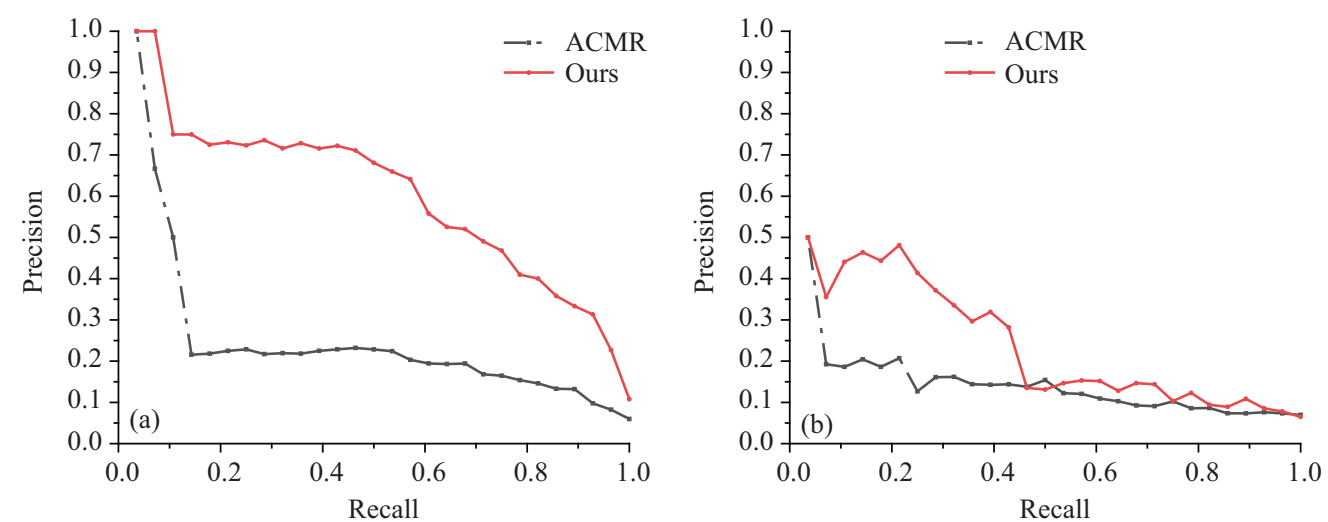

图 3 (网络版彩图) 图到文本 (a), 文本到图 (b) 搜索的 PR 曲线结果/微博数据集

Figure 3 (Color online) PR curve of image to text (a), text to image (b) search/Weibo dataset

\subsection{SSACR 方法的参数对搜索性能的影响}

SSACR 方法的主要参数是算法 1 中对抗训练中特征映射网络的训练次数 $k$ 值以及特征映射网络 的损失函数的参数, 即语义映射损失以及模态损失的权值系数 $\alpha$ 和 $\beta$.

在维基数据集和微博数据集上进行实验, 以图像搜索文本以及文本搜索图像的平均 map 值作为 评价指标, 判断 $k$ 值的对指标的影响, 实验结果分别如图 4(a) 和 (b) 所示.

本文提出的 SSACR 方法在不同数据集上 $k$ 的最优值稍有偏差, $k$ 在取 5 时达到一个较高值, 当 $k$ 值大于 5 时, map 值出现缓慢下降趋势, 因此在给定 $\alpha$ 和 $\beta$ 值的情况下, $k$ 取 5 时在当前数据集所得 map 值达到最优. 因为对抗训练中生成器的训练直接决定原始特征映射到同一语义空间的结果, 因此 在较高值下 SSACR 方法表现较优, 但是当 $k$ 过大时, 过于忽略判别器的影响导致模型数据训练不完 善, 对抗过程不充分进而导致整体表现较差.

当 $k$ 值固定时, 将 $\alpha$ 和 $\beta$ 分别从 0.1 到 100 每次放大 10 倍, 在 16 组数据中确定 $\alpha$ 和 $\beta$ 对整体 搜索效果的影响. 在维基数据集和微博数据集分别进行对比实验, 实验结果如图 5 和 6 所示. 

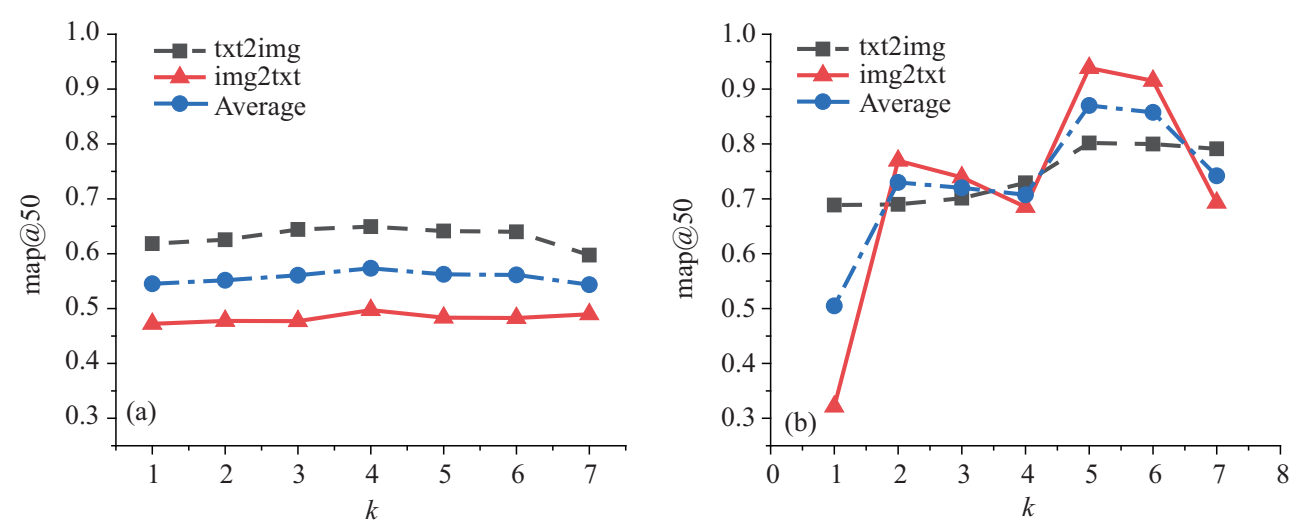

图 4 (网络版彩图) 在维基数据集 (a) 和微博数据集 (b) 上 SSACR 模型参数 $k$ 对性能的影响

Figure 4 (Color online) The performance of SSACR with different model parameter $k$ on Wikipedia dataset (a) and Weibo dataset (b)

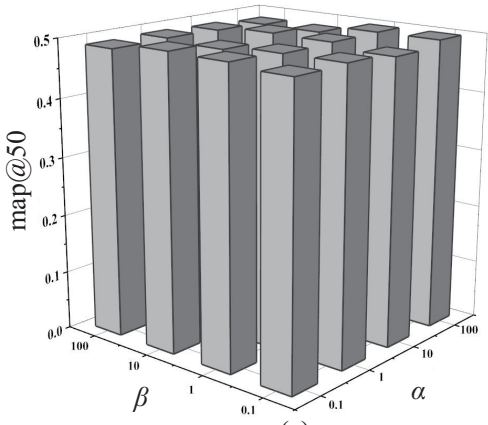

(a)

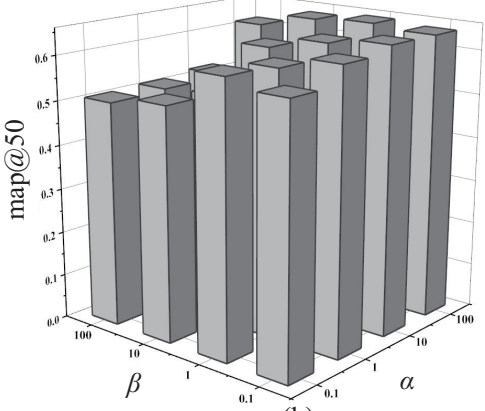

(b)

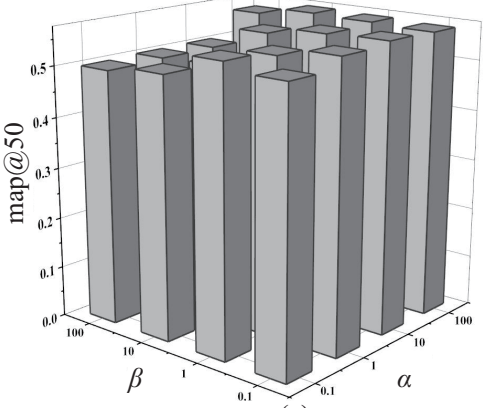

(c)

图 5 不同模型参数下的 (a) 图像到文本搜索过程影响效果, (b) 文本到图像搜索过程影响效果, (c) 平均影响效 果/维基数据集

Figure 5 (a) The performance of image to text search, (b) text to image search, and (c) the average performance with different model parameters/Wikipedia dataset

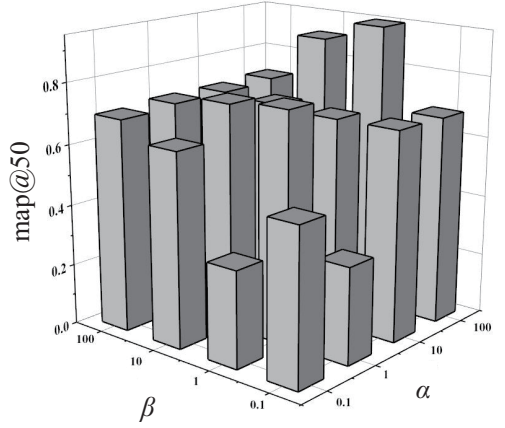

(a)

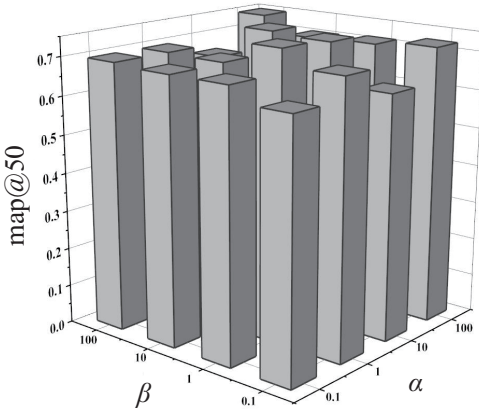

(b)

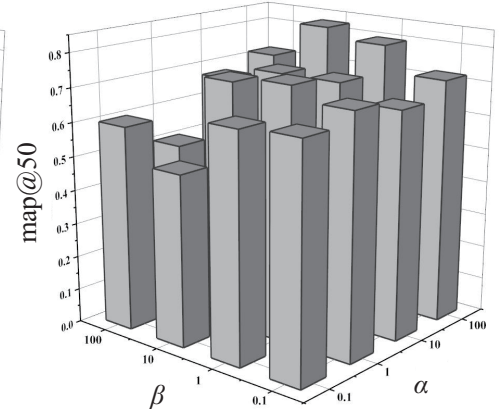

(c)

图 6 不同模型参数下的 (a) 图像到文本搜索过程影响效果, (b) 文本到图像搜索过程影响效果, (c) 平均影响效 果/微博数据集

Figure 6 (a) The performance of image to text search, (b) text to image search, and (c) the average performance with different model parameters/Weibo dataset 


\section{表 4 不同损失函数构成的 SSACR 方法在维基数据集的性能比较}

Table 4 Comparison of SSACR performance on Wikipedia dataset composed with different loss

\begin{tabular}{|c|c|c|c|c|c|c|c|c|c|}
\hline & \multicolumn{3}{|c|}{ map@5 } & \multicolumn{3}{|c|}{ map@20 } & \multicolumn{3}{|c|}{ map@50 } \\
\hline & txt2img & img2txt & Average & txt2img & img2txt & Average & txt2img & img2txt & Average \\
\hline$L_{\text {imd }}$ only & 0.6591 & 0.4859 & 0.5725 & 0.6677 & 0.4946 & 0.5811 & 0.6468 & 0.4916 & 0.5692 \\
\hline$L_{\text {imi }}$ only & 0.5118 & 0.5031 & 0.5075 & 0.5287 & 0.5055 & 0.5171 & 0.5100 & 0.4974 & 0.5037 \\
\hline Both & 0.6604 & 0.4964 & 0.5784 & 0.6647 & 0.5052 & 0.5850 & 0.6436 & 0.4964 & 0.5700 \\
\hline
\end{tabular}

表 5 不同损失函数构成的 SSACR 方法在微博数据集的性能比较

Table 5 Comparison of SSACR performance on Weibo dataset composed with different loss

\begin{tabular}{|c|c|c|c|c|c|c|c|c|c|}
\hline & \multicolumn{3}{|c|}{ map@5 } & \multicolumn{3}{|c|}{ map@20 } & \multicolumn{3}{|c|}{ map@50 } \\
\hline & txt2img & img2txt & Average & txt2img & img2txt & Average & txt2img & img2txt & Average \\
\hline$L_{\text {imd }}$ only & 0.6082 & 0.6842 & 0.6462 & 0.7094 & 0.6476 & 0.6785 & 0.7819 & 0.6577 & 0.7198 \\
\hline$L_{\mathrm{imi}}$ only & 0.6853 & 0.5031 & 0.5942 & 0.6891 & 0.5055 & 0.5973 & 0.6905 & 0.4974 & 0.5940 \\
\hline Both & 0.8792 & 0.7344 & 0.8068 & 0.8161 & 0.7361 & 0.7761 & 0.7493 & 0.7410 & 0.7452 \\
\hline
\end{tabular}

\section{5 损失函数对 SSACR 方法搜索性能的影响}

SSACR 方法的特征映射网络采用了语义偏差损失以及相似度损失进行评价. 为了说明这两个损 失函数对训练是有效的, 因此本文研究当去掉某一损失函数时对整体结果的影响. 在维基数据集以及 微博数据集进行实验, 结果分别如表 4 和 5 所示.

通过对比实验发现当不采用语义损失函数后, 维基数据集和微博数据集的 map 值均有较大下降. 当不采用相似度损失函数时, 维基数据集 map 值稍有下降, 但是微博数据集有较大的下降. 因此语义 损失函数以及模态损失函数对于整体搜索性能的影响都较大, 只有同时计算这两个损失函数, 才能完 整构建特征映射网络, 进而提高搜索性能.

epoch 和映射损失函数以及模态判别损失函数的值的关系如图 7 所示, 损失函数下降速度非常 快, 表明 SSACR 方法易于训练. 由于采用 $l_{2}$ 范数和交叉熵作为损失函数, 因此梯度连续, 训练速度 较快.

\section{6 采用 SSACR 方法实现搜索}

使用微博数据集搜索爆炸相关的文本和图像, 分别使用天津爆炸相关的微博以及和爆炸相关的图 片进行跨媒体搜索, 得到的相关搜索结果如图 8 所示.

在文本搜索图像的搜索情境下, 使用 BoW 模型对原文本的 TFIDF 特征进行提取, 经过文本特征 映射网络映射到同一语义空间. 在同一语义空间中找到图像映射后的数据, 求它们之间的相似度, 并 按相似度进行排序得到搜索结果. 图 8 中用爆炸相关文本进行搜索, 得到和爆炸灾害相关联的图像.

图像搜索文的搜索情境中, 先将图像进行大小归一化处理, 使用 VGG19 网络提取图像特征, 通过 图像特征映射网络映射到同一语义空间中. 按照相似度求得相关文本. 如图 8 所示, 根据一张爆炸相 关的图片进行搜索, 得到描述爆炸灾害的文本. 


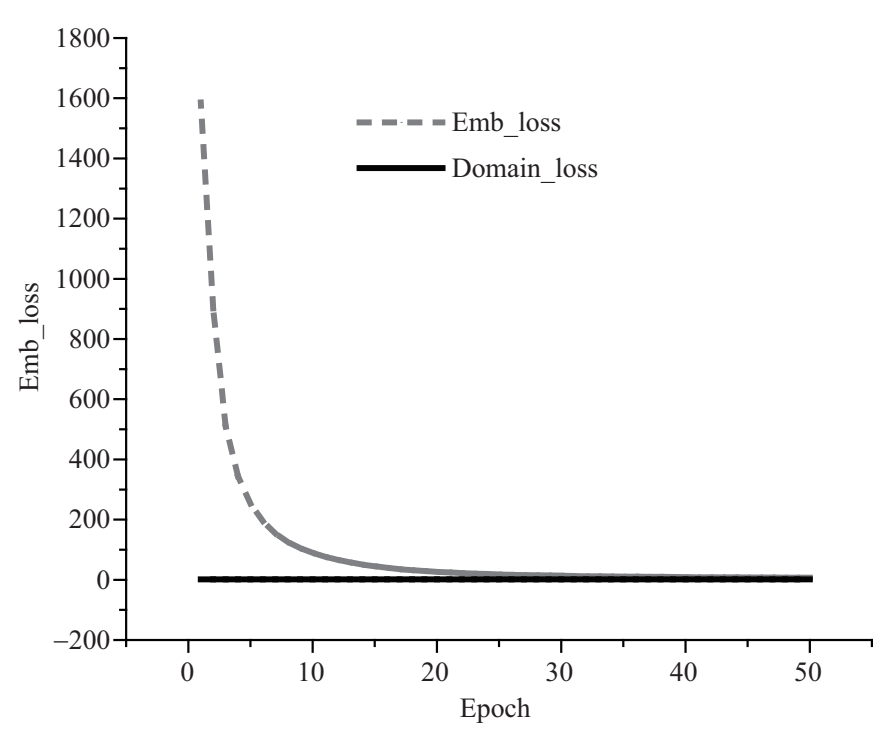

图 7 训练过程中映射损失函数和模态判别损失函数的变化

Figure 7 The embed and domain loss during the training process

Text to image
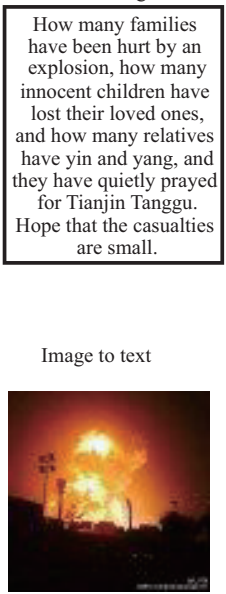

Image feature extraction
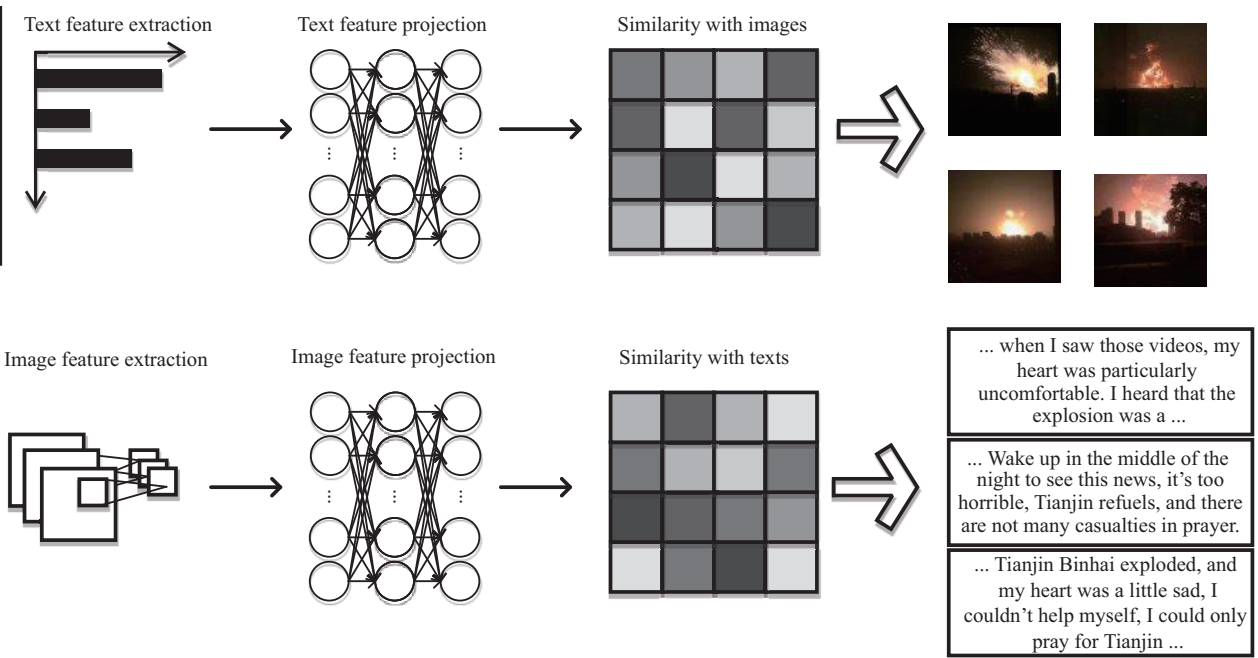

图 8 (网络版彩图) 微博数据集的跨模态搜索示例

Figure 8 (Color online) Example of cross-media retrieval on Weibo dataset

\section{6 结论}

本文提出了基于对抗学习和语义相似度的跨媒体搜索方法 (SSACR), 使用基于对抗学习的方式交 替训练特征映射网和模态判别网络, 使得跨媒体数据经过特征映射网络之后, 既保留映射前的语义分 布, 又能够消除模态上的差异, 从而达到语义相近的数据之间空间距离较近, 语义较远的数据之间空间 较远的效果. 特征映射网络使用语义分布维持映射前后数据语义分布的统一. 使用模态判别网络使得 语义映射网络映射后数据之间的模态差异保持到最小, 满足同一语义空间的性质. 结合社交网络微博 跨媒体数据的特征, 提取国民安全相关的文本和图像等跨媒体特征信息, 通过对文本以及图像进行处 
理, 将处理后的数据通过 SSACR 方法进行训练. 并根据模型得到一系列相关的跨媒体搜索结果. 本文 使用多组实验在不同数据集上验证了 SSACR 方法与其他对比方法相比具有准确率高、训练速度快等 特点. 下一步的工作是对跨媒体数据进行更多模态的映射以及研究超参数在不同数据集的偏差问题, 即如何稳定模型的超参数, 使模型更具有通用性.

\section{参考文献}

1 Bai Y. Research on tag-topic identification and community mining in social network. Dissertation for Ph.D. Degree. Dalian: Dalian University of Technology, 2018 [白杨. 社交网络中的标签主题识别及社群挖掘方法研究. 博士学位 论文. 大连: 大连理工大学, 2018]

2 Li X H, Kong W W, Ma Y Y, et al. Title microblog hot topic detection using lexical semantic co-occurrence and community partition. Appl Res Comput, 2019, 37: 1-5 [李晓红, 孔文文, 马堉垠, 等. 利用词项语义共现和社团划分 发现微博热点事件. 计算机应用研究, 2019, 37: 1-5]

3 Bai H, Lin X G. Sina weibo disaster information detection based on chinese short text classification. J Catastrophology, 2016, 31: 19-23 [白华, 林勋国. 基于中文短文本分类的社交媒体灾害事件检测系统研究. 灾害学, 2016, 31: 19-23]

4 Goodfellow I, Bengio Y, Courville A. Deep Learning. Cambridge: MIT Press, 2016

5 Goodfellow I, Pouget-Abadie J, Mirza M, et al. Generative adversarial nets. In: Proceedings of the 27th International Conference on Neural Information Processing Systems, 2014. 2672-2680

6 Radford A, Metz L, Chintala S. Unsupervised representation learning with deep convolutional generative adversarial networks. 2015. ArXiv:1511.06434

7 Li J W, Monroe W, Shi T L, et al. Adversarial learning for neural dialogue generation. 2017. ArXiv:1701.06547

8 Donahue J, Krähenbühl P, Darrell T. Adversarial feature learning. 2016. ArXiv:1605.09782

9 Wang K F, Gou C, Duan Y J, et al. Generative adversarial networks: the state of the art and beyond. Acta Autom Sin, 2017, 43: 321-332 [王坤峰, 苟超, 段艳杰, 等. 生成式对抗网络 GAN 的研究进展与展望. 自动化学报, 2017, 43: $321-332]$

10 Feng F X, Wang X J, Li R F. Cross-modal retrieval with correspondence autoencoder. In: Proceedings of the 22nd ACM International Conference on Multimedia, 2014. 7-16

11 Hardoon D R, Szedmak S, Shawe-Taylor J. Canonical correlation analysis: an overview with application to learning methods. Neural Comput, 2004, 16: 2639-2664

12 Peng Y X, Huang X, Qi J W. Cross-media shared representation by hierarchical learning with multiple deep networks. In: Proceedings of the 25th International Joint Conference on Artificial Intelligenc, 2016. 3846-3853

13 Wang K Y, He R, Wang L, et al. Joint feature selection and subspace learning for cross-modal retrieval. IEEE Trans Pattern Anal Mach Intell, 2016, 38: 2010-2023

14 Wang K Y, He R, Wang W, et al. Learning coupled feature spaces for cross-modal matching. In: Proceedings of IEEE International Conference on Computer Vision, 2013. 2088-2095

15 Li D G, Dimitrova N, Li M K, et al. Multimedia content processing through cross-modal association. In: Proceedings of the 11th ACM International Conference on Multimedia, 2003. 604-611

16 Yao T, Mei T, Ngo C W. Learning query and image similarities with ranking canonical correlation analysis. In: Proceedings of IEEE International Conference on Computer Vision, 2015. 28-36

17 Andrew G, Arora R, Bilmes J, et al. Deep canonical correlation analysis. In: Proceedings of International Conference on Machine Learning, 2013. 1247-1255

18 Zhuang Y T, Wang Y F, Wu F, et al. Supervised coupled dictionary learning with group structures for multi-modal retrieval. In: Proceedings of the 27th AAAI Conference on Artificial Intelligence, 2013

19 Ngiam J, Khosla A, Kim M, et al. Multimodal deep learning. In: Proceedings of the 28th International Conference on Machine Learning (ICML-11), 2011. 689-696

20 Yan F, Mikolajczyk K. Deep correlation for matching images and text. In: Proceedings of IEEE Conference on Computer Vision and Pattern Recognition, 2015. 3441-3450

21 Song J K, Yang Y, Yang Y, et al. Inter-media hashing for large-scale retrieval from heterogeneous data sources. In: Proceedings of ACM SIGMOD International Conference on Management of Data, 2013. 785-796 
22 Li Z C, Tang J H, Mei T. Deep collaborative embedding for social image understanding. IEEE Trans Pattern Anal Mach Intell, 2019, 41: 2070-2083

23 Wang B K, Yang Y, Xu X, et al. Adversarial cross-modal retrieval. In: Proceedings of the 25th ACM International Conference on Multimedia, 2017. 154-162

$24 \mathrm{Xu} \mathrm{X}, \mathrm{He}$ L, Lu H M, et al. Deep adversarial metric learning for cross-modal retrieval. World Wide Web, 2019, 22: $657-672$

$25 \mathrm{He} \mathrm{L}, \mathrm{Xu} \mathrm{X}, \mathrm{Lu} \mathrm{H} \mathrm{M}$, et al. Unsupervised cross-modal retrieval through adversarial learning. In: Proceedings of IEEE International Conference on Multimedia and Expo (ICME), 2017. 1153-1158

26 Li C, Deng C, Li N, et al. Self-supervised adversarial hashing networks for cross-modal retrieval. In: Proceedings of IEEE Conference on Computer Vision and Pattern Recognition, 2018. 4242-4251

27 Wu L, Wang Y, Shao L. Cycle-consistent deep generative hashing for cross-modal retrieval. IEEE Trans Image Process, 2019, 28: 1602-1612

28 Zhang J, Peng Y X, Yuan M K. Unsupervised generative adversarial cross-modal hashing. In: Proceedings of the 32nd AAAI Conference on Artificial Intelligence, 2018

$29 \mathrm{Su} \mathrm{W} \mathrm{H,} \mathrm{Yuan} \mathrm{Y,} \mathrm{Zhu} \mathrm{M.} \mathrm{A} \mathrm{relationship} \mathrm{between} \mathrm{the} \mathrm{average} \mathrm{precision} \mathrm{and} \mathrm{the} \mathrm{area} \mathrm{under} \mathrm{the} \mathrm{ROC} \mathrm{curve.}$ In: Proceedings of International Conference on the Theory of Information Retrieval, 2015. 349-352

30 Flach P, Kull M. Precision-recall-gain curves: PR analysis done right. In: Proceedings of the 28th International Conference on Neural Information Processing Systems, 2015. 838-846

31 Zhen L, Hu P, Wang X, et al. Deep supervised cross-modal retrieval. In: Proceedings of IEEE Conference on Computer Vision and Pattern Recognition, 2019. 10394-10403

\title{
A cross-media search method for social networks based on adver- sarial learning and semantic similarity
}

\author{
Chong LIU, Junping DU* \& Nan ZHOU \\ Beijing Key Lab of Intelligent Telecommunication Software and Multimedia, Beijing University of Posts and \\ Telecommunications, Beijing 100876, China \\ * Corresponding author. E-mail: junpingdu@126.com
}

\begin{abstract}
Social networks contain abundant multimedia information. The research of cross-media information search from social networks has become a hotspot. Single-modal semantic feature extractions and learnings based on deep learning show an appropriate effect on information search from social networks. But features of different modalities cannot be directly compared, therefore the semantic gap between different modalities is a key problem to be resolved for cross-media information search. Focusing on these problems, in this paper, we propose a crossmedia search method based on adversarial learning and semantic similarity to accomplish matching and sorting between text and image. This method mainly adopts adversarial learning to train a feature projecting network and a modal specific network. The feature mapping network working as a generator converts raw data from different modalities into a common semantic space based on the corresponding semantic distributions, through which the distance of data from the different modalities is small under the same semantic space while the distance of data from the same modal under different semantics is large. The generator uses semantic distributions and similarities as the basis for training the feature mapping network. The discriminator aims at distinguishing the modality of the data from different media projected into the common semantic space. The designed method with two networks is alternately trained until convergence when the features obtained by the feature mapping network are consistent with the original data semantics, and the modal feature is eliminated. Finally, the search results are obtained by similarity ranking based on the raw data from the common semantic space. The efficiency results show that the evaluation value of text and image search is higher than other similar methods. The efficiency of the method is verified on social network data on security topics.
\end{abstract}

Keywords cross-media retrieval, adversarial learning, semantic similarity, social network, search and rank 


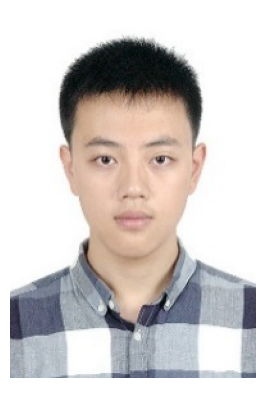

Chong LIU was born in 1995. He is a Master candidate in computer science of Beijing University of Posts and Telecommunications. His research interests include nature language processing, computer vision, and deep learning.

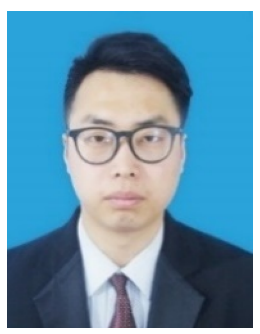

Nan ZHOU was born in 1991. Currently, he is now a Ph.D candidate in computer science of Beijing University of Posts and Telecommunications. His research interests include deep learning, information retrieval, and machine learning.

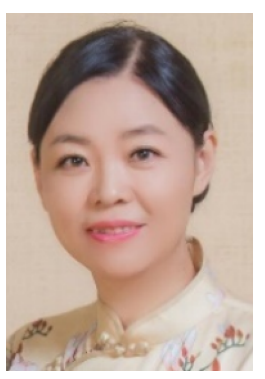

Junping DU was born in 1963. Currently, she is a professor and Ph.D. tutor at School of Computer Science and Technology, Beijing University of Posts and Telecommunications. Her research interests include artificial intelligence, image processing, and pattern recognition. 